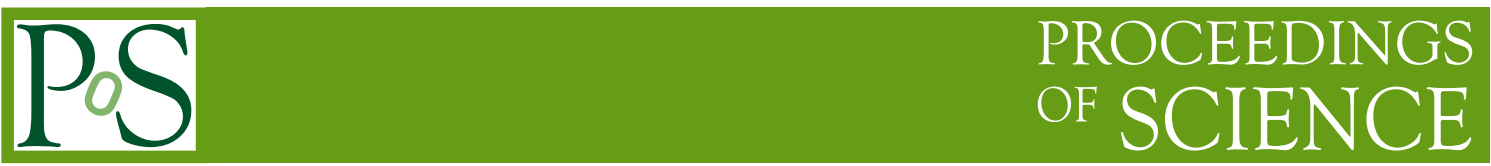

\title{
Real-time flavour tagging selection in ATLAS
}

\section{Danijela Bogavac on behalf of the ATLAS Collaboration*}

Institute of Physics, University of Belgrade, Serbia

E-mail: dbogavacecern.ch

In high-energy physics experiments, online selection is crucial to identify the few interesting collisions from the large data volume processed. In the overall ATLAS trigger strategy, $b$-jet triggers are designed to identify heavy-flavour content in real-time and, in particular, provide the only option to efficiently record events with fully hadronic final states containing $b$-jets. In doing so, two different, but related, challenges are faced. The physics goal is to optimise as far as possible the rejection of light jets from multijet processes, while retaining a high efficiency on selecting jets from beauty, and maintaining affordable trigger rates without raising jet energy thresholds. This maps into a challenging computing task, as charged-particle tracks and their corresponding vertexes must be reconstructed and analysed for each jet above the desired threshold, regardless of the increasingly harsh pile-up conditions. The performance of $b$-jet triggers during the LHC Run 1 data-taking campaigns is presented, together with an overview and preliminary results of the new online b-tagging strategy and algorithms, designed to face the above mentioned challenges, which will be adopted during Run 2 .

XXVII International Symposium on Lepton Photon Interactions at High Energies 17-22 August 2015

Ljubljana, Slovenia

\footnotetext{
*Speaker.
} 


\section{Introduction}

The ATLAS (A Toroidal LHC ApparatuS) [1] experiment at the LHC is a multipurpose detector designed to explore a wide range of physics processes including precision measurements in the context of Standard Model and searches for physics beyond the Standard Model. The trigger system of the ATLAS detector has the ability to perform real-time track reconstruction and identify jets originating from $b$-quarks ( $b$-jets). The $b$-jet triggers make it possible to collect events whose main signature is the presence of heavy flavour jets in the final state. The $b$-jet triggers are essential for many physics analyses, such as: fully hadronic $t \bar{t}$, single top, VBF $H(H \rightarrow b \bar{b})$, fully hadronic $t \bar{t} H$, supersymetric Higgs bosons produced in associations with a $b$-quark $(b A \rightarrow b b b)$, exotics signatures decaying to multi $b$-jets final state, supersymmetric signatures such as $3^{\text {rd }}$ generation squarks.

\section{The $b$-jet identification}

The identification of a jet arising from a $b$-quark ( $b$-jet) is performed taking into account the distinguishing properties of a $B$-hadron. The $B$-hadrons have sufficient lifetime ( $\sim 1.5 \mathrm{ps})$ to travel a measurable distance before decaying. This leads to the presence of a secondary vertex (SV) displaced from the primary interaction point, and of tracks with a high transverse impact-parameter. In the case of semi-leptonic $B$-hadron decays, the $b$-jets can be identified by the presence of a muon within the jet. The branching ratio of these decays is $\sim 20 \%$ including both a direct decay $b \rightarrow \mu+X$, and a cascade decay, $b \rightarrow c+X, c \rightarrow \mu+X$.

\section{ATLAS Trigger System}

The ATLAS Trigger System [2] decides which collision events are saved for offline analysis. It consists of two different types of event selection: the Level-1 (L1), and the High Level Trigger (HLT). The L1 makes a hardware-based trigger decision and uses reduced detector granularity from the calorimeters and fast muon detectors. The L1 trigger elements (jet, electromagnetic and tau clusters, muon candidate, etc.) determine Regions of Interest (RoIs) that seed further trigger decisions at the HLT. The HLT makes a trigger decision based on software algorithms running on farms equipped with commercial computing components. It can access all detectors and full granularity data. In order to deal with the increased centre-of-mass energy in Run 2, increased luminosity and number of interactions per bunch crossing, major upgrades of the trigger system were carried out during the long shutdown (2013-2015). The most important trigger hardware updates which significantly improve the b-tagging performance are described in the following.

The new L1 Topological trigger modules (L1Topo) [3] are introduced for Run 2. The L1Topo calculates event topological quantities based on more L1 objects and allows the Central Trigger Processor to perform $\mathrm{L} 1$ selections based on these quantities. These topological selections are very useful for selection of final states with $b$-jets using muons, performing a geometrical matching between muon and jet already at L1. Using this approach, these 'muon-in-jet' triggers can be used by $b$-jet trigger for physics and calibration analyses in Run 2 .

The installation of the Fast TracKer (FTK) [4] will provide the full tracking information directly after L1, allowing a fast track refit at the HLT, to improve their quality. It leads to two types 
of improvements for the $b$-jet trigger performance. A first improvement comes from the possibility to build and tag track jets instead of calorimetric jets, allowing to efficiently cover lower jet transverse momentum $p_{T}$ values. Figure 1 shows the transverse energy $E_{\mathrm{T}}$ distribution for offline b-tagged jets in simulated $t \bar{t}$ events, before and after matching with L1 calorimetric jets or track jets reconstructed using FTK.
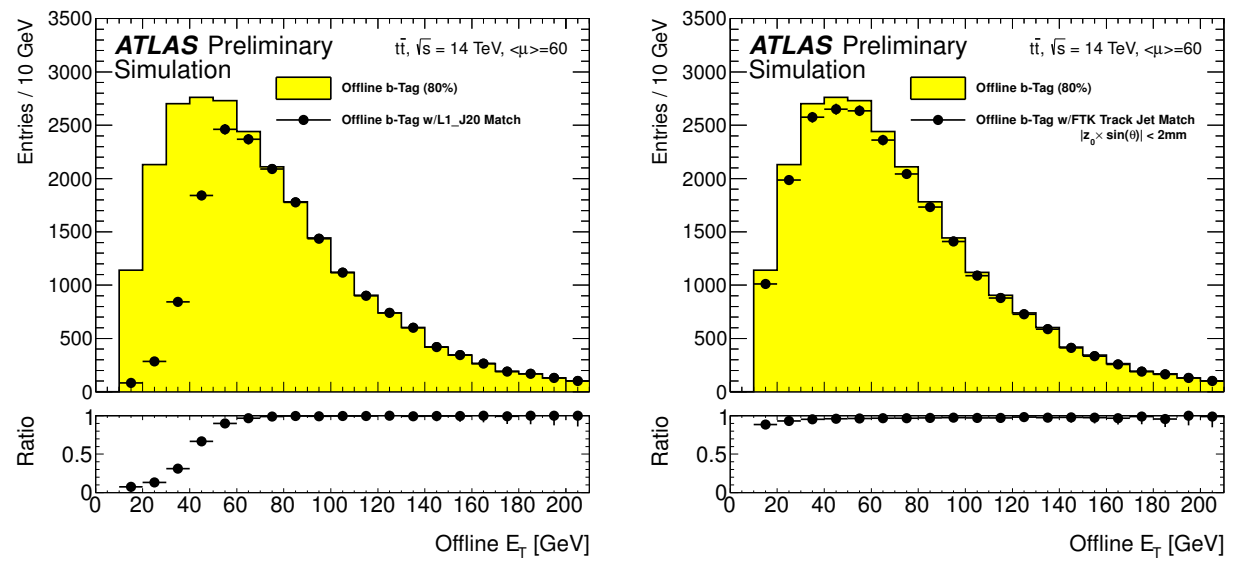

Figure 1: $E_{\mathrm{T}}$ distribution for offline b-tagged jets in simulated $t \bar{t}$ events. All jets, shown in yellow, are reconstructed using the anti-kt $(\mathrm{R}=0.4)$ algorithm and are required to have pseudorapidity $|\eta|<2.5$. The black points show the sub-set of offline jets matching a $20 \mathrm{GeV}$ L1 jet (left) or an FTK track-jet (right) [5].

Another improvement comes from the fact that faster track and vertex reconstruction will allow to run the b-tagging selections on a larger L1 input rate.

\section{Real-time b-tagging algorithms at $13 \mathrm{TeV}$}

An important improvement for Run 2 is the possibility to execute tracking and primary vertex reconstruction algorithms in one pass for all candidate jets, instead of processing them sequentially. All jet RoIs are first merged into a single, topologically unique, "Super Rol", and the overlaps of different jets within the Super RoI are removed. Fast tracking reconstruction is then performed within the entire Super RoI, to find the primary vertex of the event, exploiting tracking information from all the jets. This reduces the CPU consumption for track reconstruction by avoiding to process the same region multiple times as well as prevents track duplicates. The $b$-jet identification proceeds then individually for each jet, performing precision tracking, secondary vertex reconstruction and evaluating the $b$-jet tagging discriminant variables.

Another big improvement comes from the fact that, for Run 2, the $b$-jet trigger uses the same algorithms as the offline reconstruction. The IP3D algorithm calculates a likelihood ratio based on the two-dimensional distribution of the significance for the longitudinal and transverse impact parameters of the tracks in the jet. The SV1 algorithm exploits the secondary vertex properties such as the invariant mass of all tracks associated to the vertex, the ratio between the sum of the momenta of vertex tracks and the jet energy, and the number of two-track vertices. The JetFitter 
algorithm analyzes the topological structure of weak $b$ - and $c$-hadron decays inside the jet [6]. The variables built from these three basic algorithms are then combined. In Run 2 the combination is done using a Boosted Decision Tree (BDT). The resulting MV2 tagging algorithm is trained with different $c$-jet/light-jet proportions, e.g. MV2c20 is trained with $20 \%$ of $c$-jets and $80 \%$ of lightjets, which provides the best compromise between $c$-jet rejection and light rejection.

Some benefits of running offline algorithms online are: better performance w.r.t. Run 1 and easy harmonization of online and offline working points, because the two selections are very well correlated.

Figure 2 shows the improvements in rejection power achieved by adopting the offline algorithms for online operation.
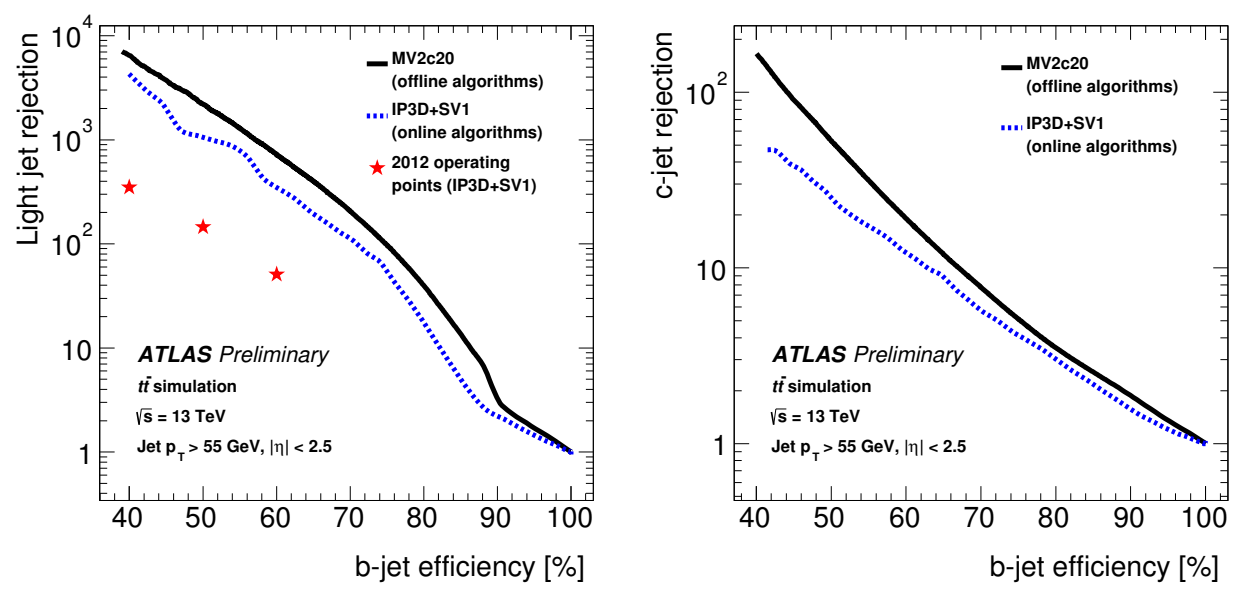

Figure 2: The expected online performance in terms of light-jet rejection (left) and c-jet rejection (right) of the MV2c20 tagger (solid black line) is shown together with the expected performance of the IP3D+SV1 tagger in Run 2 (dashed blue line) and the actual performance of the IP3D+SV1 tagger that was achieved during Run 1 (red star) [7].

\section{Summary}

The overview and preliminary results of the new online b-tagging strategy and algorithms, designed to face the new LHC Run 2 challenges are presented. These results show significant improvements in performance for Run 2.

\section{References}

[1] ATLAS Collaboration, The ATLAS Experiment at the CERN Large Hadron Collider, JINST 3 (2008) S08003.

[2] ATLAS Collaboration, Technical Design Report for the Phase-I Upgrade of the ATLAS TDAQ System, CERN-LHCC-2013-018, ATLAS-TDR-023, CERN, Geneva, December 2013.

[3] E. Simioni et al, Upgrade of the ATLAS Level-1 Trigger with event topology information, Proceedings of the CHEP2015 conference, ATL-DAQ-PROC-2015-017, J. Phys.: Conf. Ser. (2015).

[4] ATLAS Collaboration, Fast TracKer (FTK) Technical Design Report, CERN-LHCC-2013-007, ATLAS-TDR-021, CERN, Geneva, June, 2013. 
[5] ATLAS Collaboration, http://twiki.cern.ch/twiki/bin/view/AtlasPublic/FTKPublicResults.

[6] ATLAS Collaboration, ATLAS-CONF-2011-102, http://cds.cern.ch/record/1369219.

[7] ATLAS Collaboration, http://twiki.cern.ch/twiki/bin/view/AtlasPublic/BJetTriggerPublicResults. 